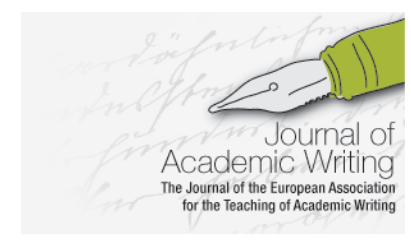

Journal of Academic Writing

Vol. 8 No 1 Summer 2018, pages ii-v http://dx.doi.org/10.18552/joaw.v8i1.530

\title{
Editorial
}

\section{Methodologies, Methods and Processes for Teaching and Assessing Academic Writing}

\author{
Welcome to the Summer 2018 issue of the Journal of Academic Writing. Before introducing \\ this issue, we would first like to announce the following new members of JoAW's editorial \\ team.
}

Dr. Mark Carver (University of St. Andrews) is our new Book Reviews Editor, and has launched into the role enthusiastically. We have two book reviews this issue and look forward to expanding JoAW's survey of current Academic Writing literature in future general issues. Mark also brings a new set of connections to the journal, and we hope to increase the breadth of JoAW's engagement with, and coverage of, the field's leading edge.

\begin{abstract}
Dr. Jia Shao and Dr. Liam Brierley from sigma, Coventry University's Mathematics and Statistics Support Service - which offers mathematics and statistics support to staff and students - now offer us statistical reviewing. Their contribution has already improved our peer review processes and we are grateful to them for lending their expertise.
\end{abstract}

Additionally, as always, we are keen to hear from potential peer reviewers, and book reviewers, as well as from publishers and authors about their recent publications. Please write to us at the relevant editorial addresses if you are willing to contribute to JoAW in these ways.

Focusing on the Summer 2018 issue, it may be helpful to note that the articles loosely group into two categories. The first set examines certain theoretical aspects of academic writing in Europe, while the second offers a range of corpora-based investigations into student writing. The issue then includes book reviews, and closes with an important invitation to readers to join in a dialogue on how colleagues currently make use of e-learning tools in their Academic Writing pedagogy and in their own academic and scholarly writing.

To open the first set of articles, Ingo Peters provides a framing analysis of the treatment of creativity in German student writing handbooks. Through a comparative analysis with US handbooks, Peters arrives at a fascinating understanding of the methodological stances behind these two distinct cultures of writing instruction. In the US, Peters finds an exploratory, inventive quality, while in Germany, texts emphasise correctness and mastery of techniques and rules. Peters' contribution extends an ongoing comparative discussion of approaches to Academic Writing pedagogy in Germany, which continues to expose interesting cultural dynamics in our field.

Complementing this study, Ursula Canton argues for a new approach to assessing the effectiveness of writing instruction. Creativity features here again, although in a different way to Peters' article. Taking inspiration from Teresa Amabile's Consensual Assessment Technique (1982) for measuring creativity, Canton grounds a method for measuring the impact Academic Writing teachers have on students' written ability. The method suggests a new way of capturing the value of our pedagogy, in terms of an Academic Literacies approach, and Canton and colleagues are already engaged in empirical testing. 
The second group of articles, which are corpora-based, begins with an ambitious study by Anna Wärnsby, Asko Kauppinen, Laura Aull, Djuddah Leijen and Joe Moxley, examining students' use of criticism and praise in peer reviewing. The corpus draws upon a substantial comparative sample of approximately 50,000 peer reviews from the University of South Florida, Malmö University and the University of Tartu. The analysis arrives at both expected and idiosyncratic findings for how students nuance their feedback with positive and negative language, and, through the discussion, opens new channels for instructional guidance on student peer reviewing, and for further investigation.

The next paper, by Ulf Olsson and David Hallberg, examines survey responses from two departments at Stockholm University. Using a quantitative approach, the research compares students' and supervisors' understanding of their responsibilities during the early stages of thesis supervision at undergraduate and taught postgraduate levels. Referring to Dysthe's three models of supervision relationships (2002), the research reveals a general consensus between students and supervisors, so leans toward the Partnership model. The results further affirm certain findings in relation to differing disciplinary understandings of particular responsibilities, but arrive at an important problem. Why do some students fail to submit on time despite this agreement of responsibilities? Olsson and Hallberg make cogent deductions from their results, and suggest ways of utilising communication in supervisory processes to better serve successful outcomes.

Tim Vandenhoek's corpus-based study examines differences in the use of epistemic markers - words showing degrees of certainty and hesitation - by native speakers (NS) and nonnative speakers (NNS) in academic writing. Vandenhoek locates the study in a sample of 'strong writing' assignments produced by a fourth-year undergraduate course at an Irish university. The investigation establishes new lists of epistemic markers and proceeds to identify a surprising trend. While NNS and NS speakers appear to show similar levels of confidence in deploying epistemic markers, NNS students' range of epistemic vocabulary and deployment of clusters of such words is far more limited, even showing particular exclusions. The research underscores a zone of linguistic competency, whereby NNS students might gain confidence and expertise in their academic writing style.

The final corpus-based study, from the University of York, demonstrates an interesting collaborative pedagogical intervention between librarians and academic staff. Focusing on the quality of Nursing students' bibliographies, David Brown, Ted Hewitt, Devi Nannen, Jessica Powell and Anita Savage Grainge suggest a correlation between the quality of references used in assignments and the overall grade of those assignments. The authors argue that the ability to critically select, appraise and organise relevant, high quality research is fundamental to both academic and professional contexts. The article rightly exhorts colleagues to introduce students to this type of critical thinking and address these skills at the earliest opportunity.

In addition to these studies, the Summer 2018 issue includes two book reviews. The first, by Steven Gump, examines Joli Jensen's Write No Matter What: Advice for Academics (2017) in light of Kristin Solli's question, in her book review published in the Summer 2018 JoAW: 'Do we need another book offering advice on academic writing?' The answer, resoundingly affirmative, confirms how guidance can be vital to researchers at any stage of their careers.

The second review, by Páraic Kerrigan and Alison Farrell, appraises editors Linda AdlerKassner and Elizabeth Wardle's Naming What We Know: Threshold Concepts of Writing Studies (2016). Beginning from a position of interconnectedness between learning, teaching and researching, a connectivity epitomised by the act of writing, Adler-Kassner and Wardle curate an encouraging consideration of how to treat writing and writing development within HE's institutional contexts. The focus on threshold concepts, according to Kerrigan and Farrell, 'provides a much needed bridge between the traditionally oppositional focuses of teaching and learning, and research'.

To close the issue, we invite JoAW's readers, through the research of Dr. Miriam Schcolnik, to engage in a 'dialogue' on how writing professionals use digital tools in their writing and their pedagogy. The initial findings of a survey conducted by Schcolnik suggest the beginnings of a 
categorisation of tools and types, opening the door to further understanding of how EATAW's community of Academic Writing teachers and scholars integrates a rapidly expanding array of software into our practice. Yet the tools discussed in the survey are only the tip of a much larger iceberg, and so we appeal to the EATAW community for further soundings.

We invite responses to Schcolnik's study that give examples of the use of digital tools for writing and writing instruction. Case studies, short investigative papers and provocations will be collated and published in a future general issue of JoAW, as outlined in our Author Guidelines ('Dialogue Responses'). A fuller invitation is framed at the end of the 'Dialogues' piece in this issue, with some suggested lines of inquiry to pursue in responses. Please, write to us if you are interested in responding.

George Ttoouli

Coventry University, UK

Lisa Ganobcsik-Williams

Coventry University, UK 


\section{References}

Amabile, T. M. (1982) 'Social Psychology of Creativity: A Consensual Assessment Technique'. Journal of Personality and Social Psychology 43 (5), 997-1013

Dysthe, O. (2002) 'Professors as Mediators of Academic Text Cultures: An Interview Study with Advisors and Master's Degree Students in Three Disciplines in a Norwegian University'. Written Communication 19 (4), 493-544. DOI: 10.1177/074108802238010 